\title{
An Enhanced Clustering Method for Extending Sensing Lifetime of Wireless Sensor Network
}

\author{
Yakubu Abdul-Wahab Nawusu ${ }^{*}$, Alhassan Abdul-Barik ${ }^{2}$ and Salifu Abdul-Mumin ${ }^{3}$ \\ ${ }^{1}$ Computer Science Department, Tamale Technical University, Tamale, Ghana \\ e-mail: nabdul-wahab@tatu.edu.gh \\ ${ }^{2}$ Computer Science Department, University for Development Studies, Tamale, Ghana \\ e-mail: abarik@uds.edu.gh \\ ${ }^{3}$ Information Systems and Technology Department, C.K. Tedam University of Technology and Applied \\ Sciences, Navrongo, Ghana \\ e-mail: asalifu@cktutas.edu.gh
}

\begin{abstract}
Extending the lifetime of a wireless sensor network is vital in ensuring continuous monitoring functions in a target environment. Many techniques have appeared that seek to achieve such prolonged sensing gains. Clustering and improved selection of cluster heads play essential roles in the performance of sensor network functions. Cluster head in a hierarchical arrangement is responsible for transmitting aggregated data from member nodes to a base station for further user-specific data processing and analysis. Minimising the quick dissipation of cluster heads energy requires a careful choice of network factors when selecting a cluster head to prolong the lifetime of a wireless sensor network. In this work, we propose a multi-criteria cluster head selection technique to extend the sensing lifetime of a heterogeneous wireless sensor network. The proposed protocol incorporates residual energy, distance, and node density in selecting a cluster head. Each factor is assigned a weight using the Rank Order Centroid based on its relative importance. Several simulation tests using MATLAB 7.5.0 (R2007b) reveal improved network lifetime and other network performance indicators, including stability and throughput, compared with popular protocols such as LEACH and the SEP. The proposed scheme will be beneficial in applications requiring reliable and stable data sensing and transmission functions.
\end{abstract}

Received: September 7, 2021; Accepted: October 13, 2021

2020 Mathematics Subject Classification: 68-XX.

Keywords and phrases: wireless sensor network, cluster head election, lifetime, throughput, stability. 


\section{Introduction}

The future is auspicious for our information gathering and processing needs. Advances in microsensor technology and collaborative micro sensing explain this promising future of intelligent data collection, processing, and fast and reliable communication. The size and cost of microsensors have decreased over the years due to advances in micro electromagnetic electronics systems (MEMS), making microsensors the preferred choice for collecting, compute and transferring data. Individually, these sensors are not robust and handy as needed. Accordingly, research focuses on collecting, processing, and reporting data from their collective interaction and cooperation. Wireless sensor network provides such collaborative data collection and processing needs. A wireless sensor network is an interconnected system of small, inexpensive, and densely deployed intelligent agents that perceive and initiate the necessary processing, fusion, and data transmission.

The physical architecture of the sensor node consists of four essential parts. The sensing subsystem performs data monitoring and converts analogue data to digital form. The subprocessor block performs minimal data processing and storage in a small memory area. The radiofrequency (RF) transceiver subsystem receives and transmits environmental messages of interest to target destinations. Finally, there is a power supply that powers the sensor node. All of these components work as a unified system to meet the specific needs of sensor network applications. There is a tracking system for some sensor nodes, such as the global positioning system, an energy harvester to convert electrical energy collected for use by the sensor node. Some sensors are designed with mobility hardware to facilitate the movement of nodes $[1,2]$.

Currently, wireless sensor networks are delivering services in many different areas of the real world. They help monitor and report to appropriate disaster management units to prevent impending devastation such as wildfires, floods, tsunamis, earthquakes, and hurricanes [1,2] and point to monitoring the strengths and weaknesses of constructions such as roads and buildings, and bridges [3,4]. The lives of human beings and animals depend mainly on the availability and purity of water sources, especially domestic water sources. WSN helps users monitor water quality and air quality.

Other uses include monitoring plant growth and animal movement, predicting the onset of disasters, assessing disaster control measures, and monitoring healthcare plans for patients and their responses, reconnaissance missions to secure homes and offices, 
and inventory and inventory counts in stores and military installations for target tracking $[3,5]$.

\section{Review of Related Literature}

Wireless sensor network deployment is either planned or unplanned. Many of its application environments require the option of random deployment of sensor nodes because of harsh and inaccessible conditions. Replacing the low-battery-powered sensor nodes in such situations is dangerous or wholly infeasible [6]. To continue to gather attribute values and information for long periods of the life of the sensor network requires efficiency in energy consumption [7]. Routing thus plays a crucial role in ensuring the longevity of a WSN. Several classes of routing protocols for WSN have appeared, including data-centric, location-based, QoS-based, and hierarchical clustering.

Classical routing protocols such as Direct Transmission (DT), Minimum Transmission Energy (MTE) yield undesirable outputs [8]. In DT, nodes directly send sensed information to the base station (BS) without recourse to intermediary nodes or specialist nodes. Nodes far away from the BS deplete their energy faster due to the increase in the transmission distance. In the case of MTE, nodes send sensed information to the BS via favourable intermediary nodes that require low transmission energy. Nodes closest to the BS in the MTE arrangement will die soonest because they will invariably serve as relay nodes to many other nodes. Again, because random deployment generates nodes close to each other, sensed data from neighbouring nodes are often correlated, causing redundancy in data sensed transmitted. MTE performs poorly in such situations. In both the DT and MTE, portions of the sensing field become unmonitored for a longer duration of the lifespan of the WSN, causing the sensing functions of the network to be biased. Data aggregation is a sure mechanism to reduce redundancy in sensor attributes. Clustering, therefore, has become an imperative paradigm for solving the problems in DT and MTE [9].

Static clustering appeared and provided a partial solution by its fixed cluster formation setup. Even though it takes away the overhead of re-clustering, it suffers because it puts an excessive burden on the cluster head, causing it to die quickest. All associated cluster members of a dead cluster head become disconnected from the base station. So their gathered data does not reach the base station, generating uneven reporting of field data. The LEACH protocol pioneered by [10] to address the concerns of static clustering allows clusters to be formed each round dynamically rather than in a 
fixed manner. LEACH implements a clustering regime to route data to a target user location - a base station. In LEACH, sensor nodes are setup into a pre-specified number of clusters. Leaders called cluster heads $(\mathrm{CH})$ are randomly selected to supervise cluster data aggregation and transmit data to the BS. Thus, non-cluster head nodes can only sense and transmit location-specific data to the associate cluster heads over a shorter distance. Many variants of the LEACH protocol have appeared in the literature that addresses the challenges of the original LEACH protocol, but many open research issues still exist.

In a heterogeneous wireless sensor network, Stable Election Protocol (SEP) [11] and its descendants including; Energy Efficient-SEP (EE-SEP), Modified Stable Election Protocol (M-SEP), Stable Election Protocol Enhanced (ESEP), Improved SEP (I-SEP), Zonal SEP (ZSEP) and Gateway Stable Election Protocol (G-SEP) have featured in research endeavours. In [12], authors modify the SEP with a new protocol called Energy Efficient-SEP (EE-SEP). The protocol succeeds in sustaining more nodes alive longer than in SEP. The network's stability, lifetime, and energy consumption bettered the performance of SEP. However, the distance was not a factor considered in the algorithm used to select the cluster head.[13] presented a multi-level hierarchical routing protocol for WSN called Modified Stable Election Protocol (M-SEP). Cluster headship is distributed, unlike in the SEP protocol. According to the authors, M-SEP outperforms SEP as to network lifespan, throughput, and energy consumption.

The scheme in [14] called Improved Stable Election Protocol (I-SEP) controls the sensing and transmission process by specifying a threshold point. Nodes are only allowed to transmit sensed attributes when it reaches a defined threshold. I-SEP introduces the third hierarchy of heterogeneity generating three node types. I-SEP does better SEP in terms of mainly increased stability period. A modified version of Enhanced Stable Election Based Routing Protocol for WSNs is proposed by[15]. Their protocol extends the Enhanced Stable Election Protocol (ESEP) by using the available energy of sensor nodes in cluster head selection. The work maximised network lifetime even without using distance information was used.

Zonal and planned system of operating the SEP appears in [16]. The resulting Zonal Stable Election Protocol (Z-SEP) divides the sensing field into three zones labelled 0, 1, and 2. Zone 0 covers the middle $60 \%$ of the sensing field, while zone 1 and zone 2 respectively cover the bottom $20 \%$ and top $20 \%$ of the sensing field. By design, normal nodes are generated randomly into zone 1, transmitting directly to the base station. 
Advanced nodes are deployed randomly in zone 1 and 2. Clustering then enables data transmission in those zones to the base station via an elected cluster head. The advance node on the border atop and beneath zone 0 do not participate in the cluster formation in zone 1 and 2; instead, they transmit their data outright to the base station. Z-SEP enhances SEP in many respects, but like in SEP fails to consider node distance and node neighbours density in cluster head selection.[17] presented a Gateway-SEP protocol that uses nodes' distance and residual energy in selecting cluster heads in pretty recent research.

Additionally, their protocol, G-SEP, introduces a gateway node positioned in the middle of the sensing field, allowing the positioning of the BS far away. The authors endeavoured to solve the nonconformance of the original SEP protocol to having the BS positioned outside the sensing field. G-SEP performs better than the Z-SEP in metrics like stability period, throughput, and network lifetime. However, careful consideration of the cost of the Gateway nodes is not done and evaluated. Because sensors are generally costly, it will be worthwhile to examine other cheaper means to extend the lifespan of a sensor network without introducing (expensive) new nodes. In Z-SEP, the sensor nodes are deployed in a planned manner, and consequently, the proposal in [17] is arranged in the same fashion to enable comparison. In this work, a comparatively cheaper, multicriteria cluster head selection technique has been proposed and applied in offering an extended lifespans solution to the operations of a heterogeneous wireless sensor network. The combined factors, residual energy, distance, and node burden - community size - are used in electing a leader for a cluster on a rotational basis.

\section{Methodology}

The proposed multi-criteria cluster head selection algorithm is present here, along with simulation results and discussion.

\subsection{Proposed cluster head selection approach}

Many of the SEP protocol problems arise because of the randomness in the cluster head selection algorithm. In the original SEP, no node characteristics decide its cluster head chance. However, network performance is improved when enhancement in the cluster head selection criterion incorporates other important node characteristics. In this work, three important node attributes - residual energy (remaining energy of node), node density (number of neighbours), and distance (between nodes and base station) are all considered in the cluster head selection algorithm. Each of these factors is defined below; 
Energy Ratio: Message transmission is mainly dependent on the availability of energy in a sensor node. Therefore, non-CH nodes with the maximum Energy Ratio each round are favoured to become cluster heads. For each sensor node $i$ in the network, this ratio is denoted as;

$$
\frac{E(i)}{E_{o}}
$$

where $E(i)$ is the residual energy of a given node and, $E_{o}$ is the initial energy of nodes at the start of the deployment.

Node Distance Ratio: The distance between a given node and the base station and the maximum distance between nodes still alive in the network and the base station. For each node $i$, the node distance ratio to the base station is given as;

$$
\frac{D_{t o B S}(i)-\min D_{t o B S}}{\max D_{t o B S}-\min D_{t o B S}} \text {. }
$$

Node Density: This is the ratio of the number of nodes served by a candidate cluster head to the number of nodes alive during the selection round. The node density is given as;

$$
\operatorname{Node}_{N b}(i) \text {. }
$$

Nodes with a higher value Node Density Ratio are favoured next for cluster headship. Based on the three criteria above, we computer the fitness function for each node $i$ ( $i$ is either a normal node or advance node) as expressed below;

$$
f(i)=\omega_{1} \times \frac{E(i)}{E_{0}}+\omega_{2} \times \frac{D_{t o B S}(i)-\min D_{t o B S}}{\max D_{t o B S}-\min D_{t o B S}}+\omega_{3} \times N o d e_{N b}(i),
$$

where $0<\omega_{3}<\omega_{2}<\omega_{1}<1$ areweights assigned the factors in Equations (1), (2), and (3), respectively.

There are several multi-criteria rank-weighting methods in the literature for converting criteria ranks into weights. In this work, we use the Rank Order Centroid (ROC) procedure [18] given in Equation (5). The strength of the ROC method is that the error from ranking criteria is much less and its distributions are statistically comparable to algorithmic approaches of experts.

$$
\omega_{i}(R O C)=\frac{1}{c} \sum_{i=1}^{c}\left(\frac{1}{i}\right),
$$

where $c$ is the number of criteria and, $\omega_{i}$ represents the weights of the different criteria. 
A definition for a new cluster head election threshold for normal nodes and advance nodes is derived below, respectively, considering the fitness function in Equation (4).

$$
\mathrm{T}(n r m)= \begin{cases}\frac{p_{n r m}}{\left(1-p_{n r m}\right) \times\left(\operatorname{rmod}\left(\frac{1}{p_{n r m}}\right)\right)} \times f(n r m) & n r m \in G, \\ 0 & \text { otherwise, }\end{cases}
$$

where; $r$ is the current round, $G$, is the set of normal nodes that have not been cluster heads in the last $1 / p_{n r m}$ rounds, $f(n r m)$ is the cost function for normal nodes, and $\mathrm{T}(\mathrm{nrm})$ is the threshold defined for the normal nodes.

$$
\mathrm{T}(a d v)= \begin{cases}\frac{p_{a d v}}{\left(1-p_{a d v}\right) \times\left(\operatorname{rmod}\left(\frac{1}{p_{a d v}}\right)\right)} \times f(a d v) & a d v \in G_{\prime \prime} \\ 0 & \text { otherwise, }\end{cases}
$$

where; $r$ is the current round, $G_{\prime \prime}$ is the set of advanced nodes that have not been cluster heads in the last $1 / p_{p_{a d v}}$ rounds and $f(a d v)$ is the cost function for advance nodes, $\mathrm{T}(a d v)$ is the threshold defined for the advance nodes. Figure 2 shows the cluster head selection approach proposed in this work.

\subsection{Energy model}

The radio energy consumption model in [10] depicted in Figure 1 is adopted to analyse the proposed model.

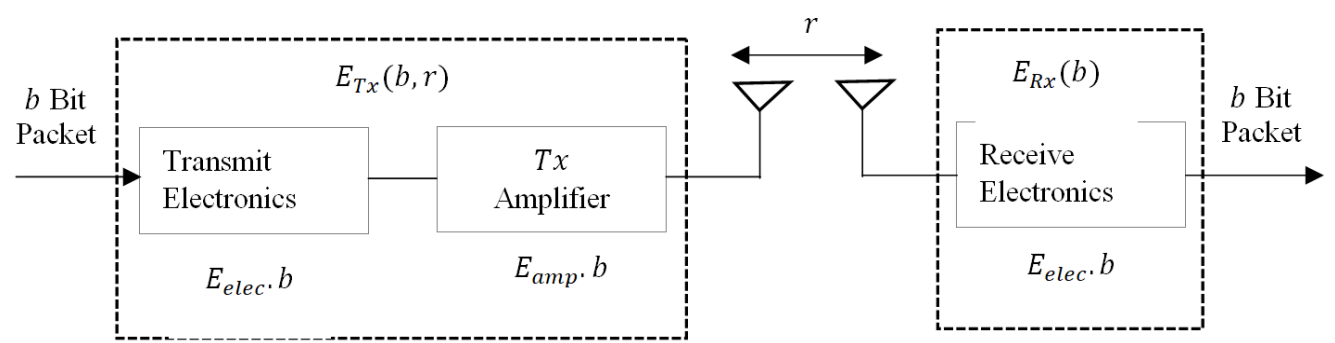

Figure 1: Radio energy model for wireless sensor network.

The transmitter component expends energy to operate both the transmit electronics and the radio circuitry, whiles the receiver component only expends energy to run the radio circuitry. Therefore, the power used up to transfer a $b$ bit message from a sending node to a receiving node which is $r$ meters apart is given as: 


$$
\begin{gathered}
E_{T x}(b, r)=b . E_{\text {elect }}+b \cdot E_{a m p} \\
E_{R x}(b, r)=b . E_{\text {elect }},
\end{gathered}
$$

where $E_{T x}$ represents the total energy consumed per bit in transmitting from the source node and $E_{R x}$ the total energy consumed in the receiver unit to receive $b$ bit of data (message). Data transmission requires additional energy to amplify the signal; this extra energy is dependent on how far apart the sending and receiving nodes are from each other. The distance is represented as $E_{a m p}$ from Equation (8) above. For short distance transmissions, i.e., free space model, $E_{a m p}$ is given as $\varepsilon_{f s} \cdot r^{2}$. Whiles for multi-path model, $E_{a m p}$ is given as $\varepsilon_{m p} \cdot r^{4}$. Therefore Equation (8) can be restated as:

$$
E_{T X}(b, r)=\left\{\begin{array}{l}
b \cdot E_{\text {elect }}+b \cdot \varepsilon_{f s} \cdot r^{2}, \quad \text { andif } r \leq r_{o} \\
b . E_{\text {elect }}+b \cdot \varepsilon_{m p} \cdot r^{4}, \quad \text { andif } r>r_{o}
\end{array}\right.
$$

where $r_{o}$ is the threshold distance derived by equating the two expressions at $r=r_{o}$ and it is given as;

$$
r_{o}=\sqrt{\frac{\varepsilon_{f s}}{\varepsilon_{m p}}} .
$$

\subsection{Network assumptions}

The following assumptions are in effect in respect of the proposed approach.

1. The sensing field is $100 \mathrm{~m} \times 100 \mathrm{~m}$ in dimension and contains 100 sensor nodes.

2. The base station is fixed and positioned at the centre of the sensing field - at $50 \mathrm{~m} \times 50 \mathrm{~m}$.

3. The base station has unlimited energy capacity.

4. All other nodes are randomly distributed and stationary.

5. $m(=10 \%)$ of the deployed nodes have extra energy than the remaining nodes.

6. Nodes are given a unique ID for identification purposes.

7. The energy model proposed in Heinzelman et al. [10] is adopted. 


\subsection{The radio characteristics for simulation}

Table I summarises the radio characteristics for simulations.

Table I. Characteristics of the 100-node simulated wireless sensor network.

\begin{tabular}{|l|l|}
\hline Operation & Energy requirement \\
\hline Transmitter/Receiver Electronics & $E_{\text {elec }}=50 \mathrm{~nJ} / \mathrm{bit}$ \\
\hline Data Aggregation & $E_{D A}=5 \mathrm{~nJ} / \mathrm{bit} / \mathrm{packet}$ \\
\hline Transmit amplifier for $r \leq r_{o}$ & $\varepsilon_{f s}=10 \mathrm{pJ} / \mathrm{bit} / \mathrm{m}^{2}$ \\
\hline Transmit amplifier for $r>r_{o}$ & $\varepsilon_{m p}=0.0013 \mathrm{pJ} / \mathrm{bit} / \mathrm{m}^{4}$ \\
\hline Size of sensing field & $100 \mathrm{~m} \times 100 \mathrm{~m}$ \\
\hline Sink node position & $50 \mathrm{~m} \times 50 \mathrm{~m}(\mathrm{center})$ \\
\hline Number of nodes $(n)$ & 100 \\
\hline Packet size & 4000 \\
\hline The initial energy of normal nodes & $0.5 \mathrm{~J}$ \\
\hline
\end{tabular}

\subsection{Performance measures}

For purposes of evaluating the suitability and effectiveness of the proposed solutions to the research questions, the following performance metrics are of interest:

Throughput: a measure of the total amount of sensed environmental attribute (s) that reaches the base station from the application areas for the entire life of the network.

Network Lifespan: the functional period of the network, marked by the time interval between the time of deployment and the death of the last alive node.

Stability Period: the period between network deployment and the exhaustion of the energy of the first sensor node.

The NumberofNodesAlive: a measure of the number of nodes that have not yet dissipated all of their energy.

The NumberofDeadNodes: the per round number of nodes that have dispersed all of its' battery energy. 


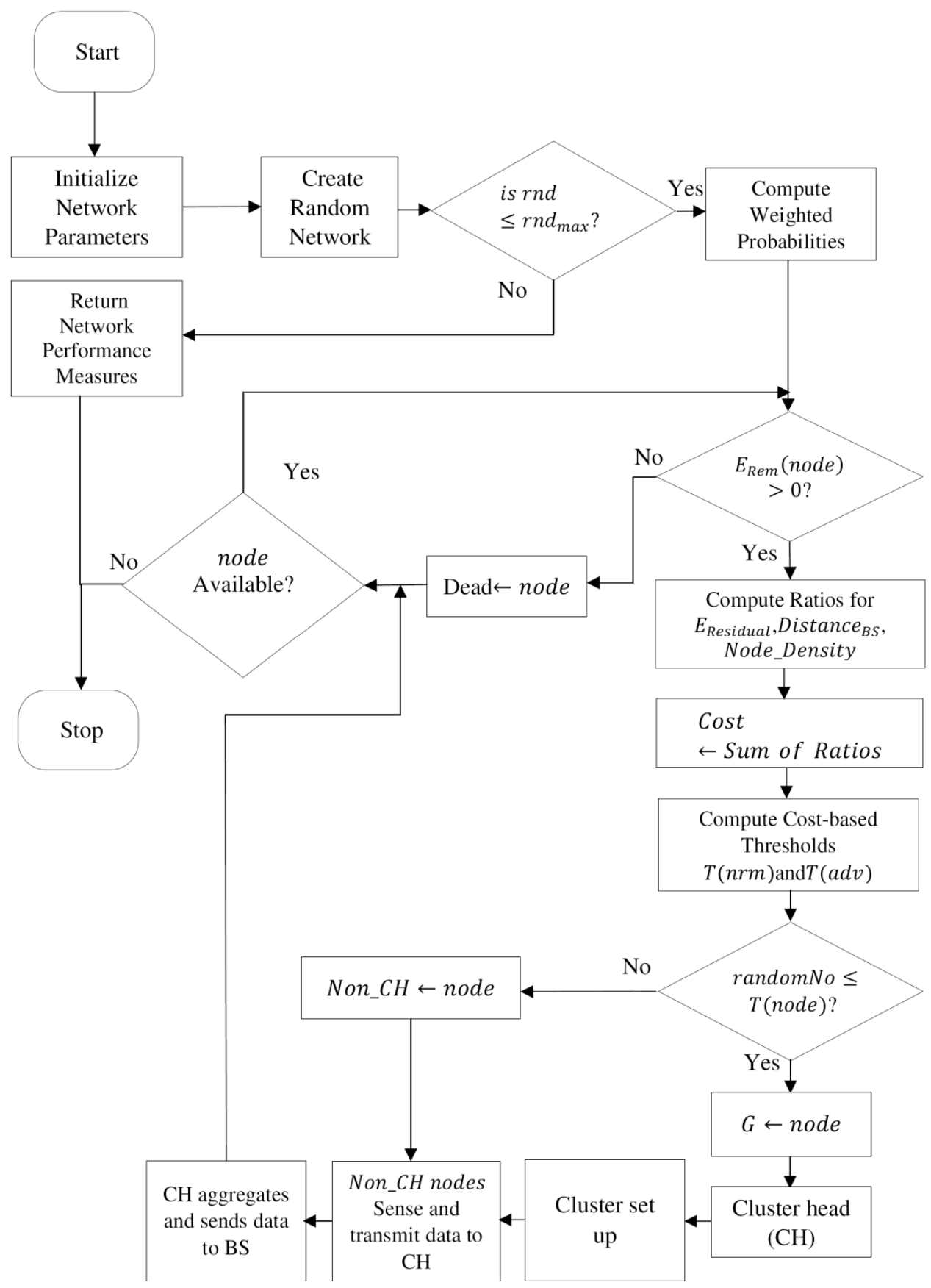

Figure 2: The flowchart for the proposed cluster selection approach. 


\section{Simulation Results and Discussion}

MATLAB 7.5.0 (R2007b) is used to simulate the sensor network and generate critical data and performance characteristics. The analysis compares results from the proposed approach in this work and the original SEP protocol using the performance metrics indicated in section 3.5. Figure 3 is the network of 100 sensor nodes randomly distributed over a 100 x 100 sensing field. The symbol 'o' (in blue) signifies normal nodes. In contrast, ' + ' (in red) indicates advanced nodes, which constitute $2 \%$ of the entire deployment and have $\alpha=1$ more energy than the initial energy of normal nodes. The BS (marked '*' (in blue)), is at the centre of the field as in the original SEP protocol.

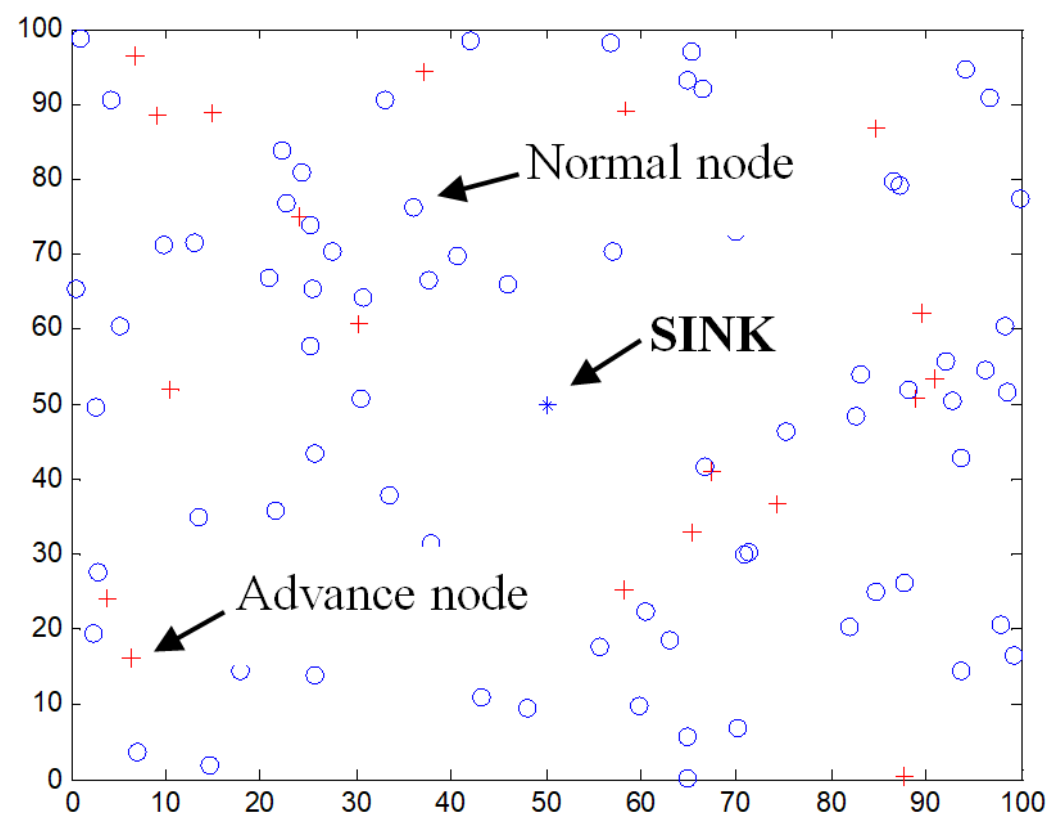

Figure 3: Network of 100 randomly distributed sensor nodes.

Figure 4 shows the number of alive nodes in each round (of 10000 rounds). It indicates the stability of the protocols. SEP and the enhanced version of SEP are better than LEACH because of the oblivious nature of LEACH to heterogeneity, which makes nodes die early. It is clear also that the proposed approach is far more stable than SEP by as much as $29 \%$. Essentially, this is because of the improvement in the cluster headship selection compared to the randomised weighted probabilities used in the original SEP. 


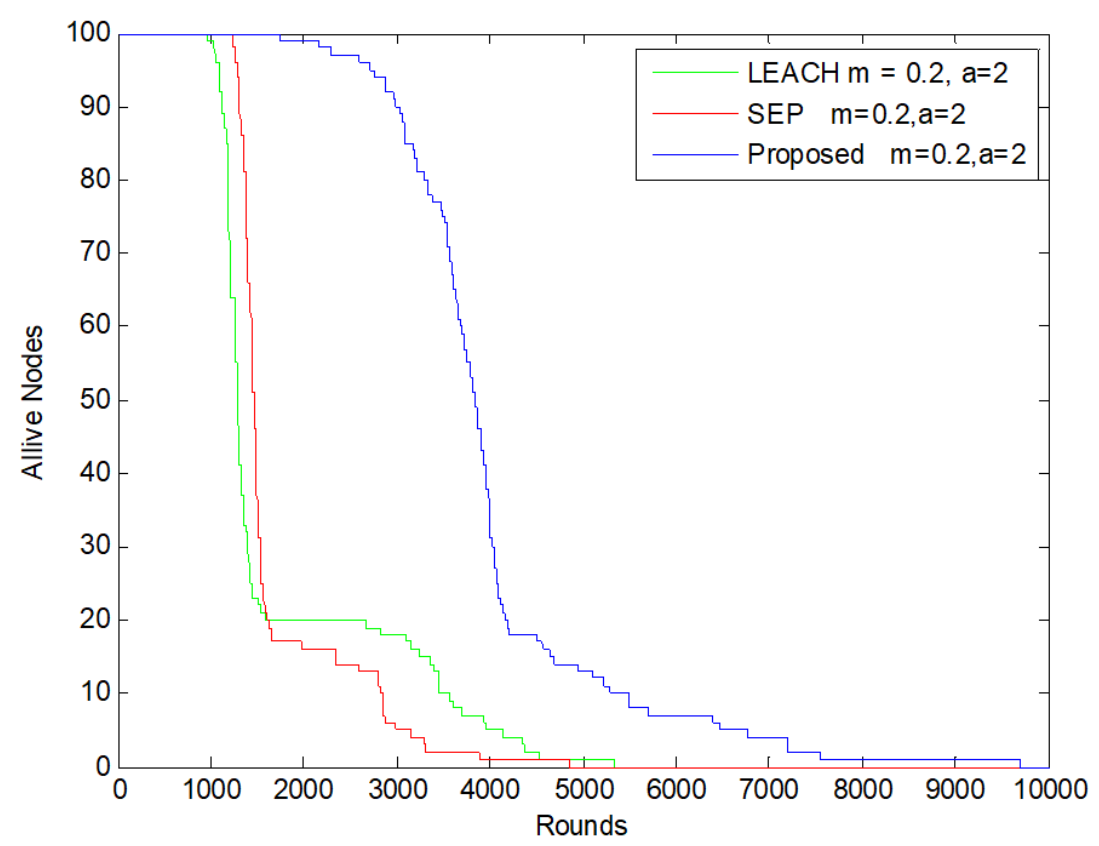

Figure 4: Number of alive nodes per round in LEACH, SEP, and Proposed scheme.

The number of dead nodes per round (10000 rounds) in the network is as in Figure 5. It is clear from the depiction in Figure 5 that the proposed method extends the duration elapsed before the death of the first node. The first node dies after 957 rounds in LEACH, whiles the first node dies after 1228 rounds in SEP. The new approach increases the dead duration for the first node by 510 rounds. At least a single node exists up to about the 1800th round in the proposed scheme. The last node dies in rounds 5300 and 4800 in LEACH and SEP, respectively.

Another measure of performance of a sensor network is the throughput - the number of packets sent to the base station or delivered to the target user. Figure 6 compares the network throughput in LEACH, SEP, and the new selection approach of cluster heads. Again, the overall throughput of the new clustering method is far greater than that of SEP, whiles SEP, in turn, is greater than that of LEACH. 


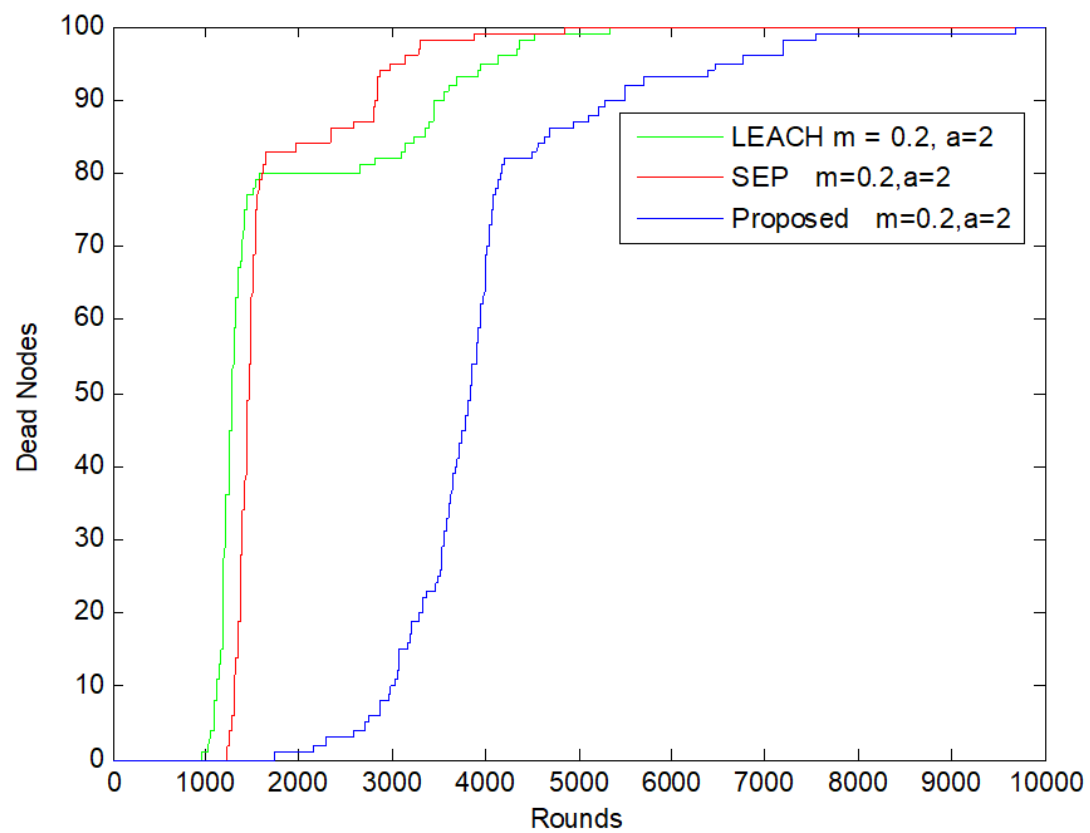

Figure 5: Number of dead nodes per round in LEACH, SEP, and Proposed scheme.

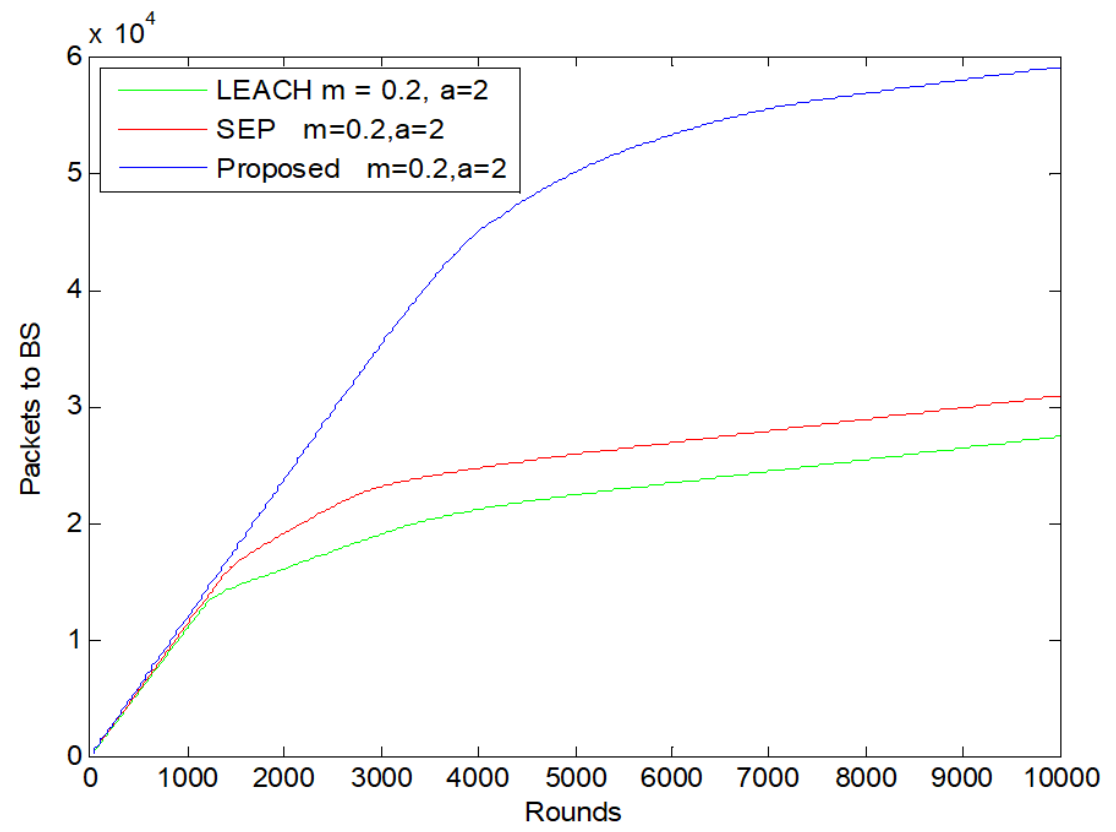

Figure 6: Throughput of the network in LEACH, SEP, and Proposed scheme. 
Table II compares average results from LEACH, SEP, and the new cost-based approach of selecting clusters. The proposed protocol enjoys a nearly 39\% stability period increase from LEACH and SEP. Similarly, network lifetime and throughput increase by approximately $54 \%$ and $44 \%$ when the proposed approach is used compared to SEP and LEACH, respectively.

Table II: A comparison of LEACH, SEP, and proposed approach $(m=0.2, a=1)$.

\begin{tabular}{|l|l|l|l|}
\hline Protocol & $\begin{array}{l}\text { Stability Period } \\
\text { (Rounds) }\end{array}$ & $\begin{array}{l}\text { Network lifespan } \\
\text { (Rounds) }\end{array}$ & $\begin{array}{l}\text { Network Throughput } \\
\text { (Packets) }\end{array}$ \\
\hline \hline LEACH & 1022 & 2248 & $2.4922 \times 10^{4}$ \\
\hline SEP & 1105 & 1677 & $2.5049 \times 10^{4}$ \\
\hline $\begin{array}{l}\text { Proposed } \\
\text { approach }\end{array}$ & 1731 & 5456 & $5.4507 \times 10^{4}$ \\
\hline
\end{tabular}

Table III: A comparison of LEACH, SEP, and proposed approach $(m=0.2, a=2)$.

\begin{tabular}{|l|l|l|l|}
\hline Protocol & $\begin{array}{l}\text { Stability Period } \\
\text { (Rounds) }\end{array}$ & $\begin{array}{l}\text { Network lifespan } \\
\text { (Rounds) }\end{array}$ & $\begin{array}{l}\text { Network Throughput } \\
\text { (Packets) }\end{array}$ \\
\hline \hline LEACH & 1031 & 3549 & $2.7555 \times 10^{4}$ \\
\hline SEP & 1218 & 2557 & $3.0500 \times 10^{4}$ \\
\hline Proposed approach & 1989 & 5605 & $5.4847 \times 10^{4}$ \\
\hline
\end{tabular}

\section{Conclusion}

Optimising the battery life of sensor nodes is still a challenging task for researchers in WSN. Cluster head selection plays crucial importance in the efficient utilisation of energy in a wireless sensor network. In this work, we propose a new approach to cluster head selection to enhance the Stable Election Protocol (SEP). The method uses a multicriteria technique that considers three factors - residual energy of node, node neighbour density, and distance to the base station - with different weights in electing cluster heads. The original SEP does not use these parameters in their combined form. Several simulations run using MATLAB 7.5.0 (R2007b) reveal that the proposed approach outperforms LEACH and SEP protocols in stability, network lifetime, and throughput. As a future extension, we will seek improvement in network performance by deducing an optimal base station location. 


\section{References}

[1] K. S. Adu-Manu, N. Adam, C. Tapparello, H. Ayatollahi and W. Heinzelman, Energyharvesting wireless sensor networks (EH-WSNs): A review, ACM Transactions on Sensor Networks 14(2) (2018), 1-50. https://doi.org/10.1145/3183338

[2] I. F. Akyildiz, W. Su, Y. Sankarasubramaniam and E. Cayirci, Wireless sensor networks: a survey, Computer Networks 38 (2002), 393-422.

https://doi.org/10.1016/S1389-1286(01)00302-4

[3] S. Kim, S. Pakzad, D. Culler, J. Demmel, G. Fenves, S. Glaser and M. Turon, Health monitoring of civil infrastructures using wireless sensor networks, IPSN'07: Proceedings of the 6th International Conference on Information Processing in Sensor Networks, 2007, pp. 254-263. https://doi.org/10.1145/1236360.1236395

[4] X. Hu, B. Wang and H. Ji, A wireless sensor network-based structural health monitoring system for highway bridges, Computer-Aided Civil and Infrastructure Engineering 28(3) (2013), 193-209. https://doi.org/10.1111/j.1467-8667.2012.00781.x

[5] S. El Khediri, N. Nasri, A. Wei and A. Kachori, A new approach for clustering in wireless sensors networks based on LEACH, Procedia Computer Science 32 (2014), 1180-1185. https://doi.org/10.1016/j.procs.2014.05.551

[6] H. Gou and Y. Yoo, An energy balancing LEACH algorithm for wireless sensor networks, Seventh International Conference on Information Technology: New Generations, 2010, pp. 822-827. https://doi.org/10.1109/ITNG.2010.12

[7] S. Lindsey and C. S. Raghavendra, PEGASIS: Power-efficient gathering in sensor information systems, Proceedings, IEEE Aerospace Conference, 2002. https://doi.org/10.1109/AERO.2002.1035242

[8] S. D. Muruganathan, D. C. F. Ma, R. I. Bhasin and A. O. Fapojuwo, A centralised energyefficient routing protocol for wireless sensor networks, IEEE Communications Magazine 43(3) (2005), 8-13. https://doi.org/10.1109/mcom.2005.1404592

[9] A. A. Abbasi and M. Younis, A survey on clustering algorithms for wireless sensor networks, Computer Communications 30 (2007), 2826-2841.

https://doi.org/10.1016/j.comcom.2007.05.024

[10] W. R. Heinzelman, A. Chandrakasan and H. Balakrishnan, Energy-efficient communication protocol for wireless microsensor networks, Proceedings of the 33rd Annual Hawaii International Conference on System Sciences, 2000.

https://doi.org/10.1109/hicss.2000.926982 
[11] G. Smaragdakis, I. Matta and A. Bestavros, SEP: A stable election protocol for clustered heterogeneous wireless sensor networks, Journal of Innovative Research in Computer and Communication Engineering 4(6) (2004), 54-59.

[12] C. Divya, N. Krishnan and T. Gandhi, Energy efficient stable election protocol for clustered heterogeneous wireless sensor networks, IOSR Journal of Computer Engineering (IOSR-JCE) 12(5) (2013), 55-61. https://doi.org/10.9790/0661-1255561

[13] G. Arya and D. S. Chauhan, Modified stable election protocol (M-SEP) for hierarchical WSN, International Journal of Computer Applications 79(16) (2013), 35-39. https://doi.org/10.5120/13947-1926

[14] R. Verma, P. Dolly and D. Kumar, A novel approach of improved stable election protocol (I-SEP) in WSN, International Journal of Innovative Research in Computer and Communication Engineering 4(6) (2015), 54-59.

[15] L. J. P. Jeevan and D. H. Manjaiah, Modified enhanced stable election based routing protocol for wireless sensor networks, International Journal of Innovative Research in Computer and Communication Engineering 2(5) (2014), 54-59.

[16] S. Faisal, N. Javaid, A. Javaid, M. A. Khan, S. H. Bouk and Z. A. Khan, Z-SEP: Zonalstable election protocol for wireless sensor networks, 2013. http://arxiv.org/abs/1303.5364

[17] F. Jibreel, M. I. Daabo and K. A. Gbolagade, Gateway-stable election protocol for heterogeneous wireless sensor network, Asian Journal of Research in Computer Science 5(1) (2020), 40-48. https://doi.org/10.9734/ajrcos/2020/v5i130127

[18] E. Roszkowska, Rank ordering criteria weighting methods - a comparative overview, Optimum. Studia Ekonomiczne 5(65) (2013), 14-33.

https://doi.org/10.15290/ose.2013.05.65.02

This is an open access article distributed under the terms of the Creative Commons Attribution License (http://creativecommons.org/licenses/by/4.0/), which permits unrestricted, use, distribution and reproduction in any medium, or format for any purpose, even commercially provided the work is properly cited. 